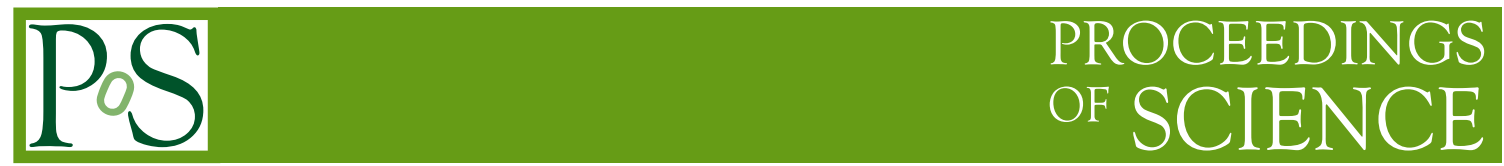

\title{
Group structure of the integration-by-part identities
}

\section{Roman N. Lee}

Budker Institute of Nuclear Physics

and

Novosibirsk State University,

630090 Novosibirsk, Russia

E-mail: r.n.leedinp.nsk.su

The problem of the IBP reduction is reformulated as that of reduction with respect to the direct sum of definite left and right ideals. Lie-algebraic properties of the IBP identities are discussed. The application of the criteria obtained in Ref. [8] is demonstrated.

XII Advanced Computing and Analysis Techniques in Physics Research November 3-7 2008

Erice, Italy 


\section{Introduction}

Multiloop calculations are important from both theoretical and practical points of view. There is a number of mathematical techniques which are used for such calculations. Some techniques, e.g., Feynman parameterization and Mellin-Barnes representation, are aimed at the direct calculation of a given diagram. However, these techniques are not always successful when applied to the diagrams with large number of the internal and/or external lines. A more universal approach is the reduction of a given loop integral to some number of the master integrals. The master integrals may then be evaluated using the above techniques. Very important advantage of the reductionbased approach is that it provides new methods of the evaluation of the master integrals. Namely, the reduction procedure allows one to obtain the linear differential/difference equations for the master integrals with respect to some continuous/discrete parameter (external invariant, denominator power, or space-time dimensionality). Solving these equations is often simpler than the direct calculation of the master integrals.

The first step of the whole reduction procedure is the tensor reduction which expresses the tensor integrals via the scalar integrals. This step is well understood and can be performed using modern computer programs. After this, one is left with the integrals of the form

$$
J(\mathbf{n})=J\left(n_{1}, n_{2}, \ldots, n_{N}\right)=\int d^{\mathscr{D}} l_{1} \ldots d^{\mathscr{D}} l_{L} j(\mathbf{n})=\int \frac{d^{\mathscr{D}} l_{1} \ldots d^{\mathscr{D}} l_{L}}{D_{1}^{n_{1}} D_{2}^{n_{2}} \ldots D_{N}^{n_{N}}},
$$

where the scalar functions $D_{\alpha}$ are linear polynomials with respect to $s_{i j}=l_{i} \cdot q_{j}$ ( $l_{i}$ is a loop momentum, $q_{j}$ is either loop or external momentum). The functions $D_{\alpha}$ are assumed to be linearly independent and to form a complete basis in the sense that any non-zero linear combination of them depends on the loop momenta, and any $s_{i j}$ can be expressed in terms of $D_{\alpha}$.

Basic idea of further reduction is to use some identities between the integrals with different multiindex $\mathbf{n}$ in order to express the complex integrals via the simpler ones. For the reduction procedure to work, it is necessary to define some suitable ordering of the integrals, i.e., the ordering in $\mathbb{Z}^{N}$. First, one introduces the notion of sectors in $\mathbb{Z}^{N}$. The $\boldsymbol{\theta}=\left(\theta_{1}, \ldots, \theta_{N}\right)$ sector, where $\theta_{i}=$ 0,1 , is a set of all points $\left(n_{1}, \ldots, n_{N}\right)$ in $\mathbb{Z}^{N}$ whose coordinates obey the condition $\operatorname{sign}\left(n_{\alpha}-1 / 2\right)=$ $2 \theta_{\alpha}-1$. In particular, the point $\left(\theta_{1}, \ldots, \theta_{N}\right)$ belongs to the $\left(\theta_{1}, \ldots, \theta_{N}\right)$ sector, and can be referred to as the corner point of the sector. Owing to this definition, the integrals of the same sector have the same set of denominators. It is natural to consider the integrals with less denominators to be simpler. When the number of denominators coincides, we will consider the integrals with smaller total power of the numerators and denominators to be simpler. Then goes the total power of the numerators and the last is the lexicographical ordering.

Among the identities which can be used for the reduction are the symmetry relations and integration-by-part identities. Symmetry relations reflect the symmetries of the original diagram and of the diagrams with some lines contracted. The IBP identities [1,2] come from the fact that, in dimensional regularization, the integral of the total derivative is zero.They are derived from the identity

$$
0=\int d^{\mathscr{D}} l_{1} \ldots d^{\mathscr{D}} l_{L} O_{i k} j(\mathbf{n})
$$

where $O_{i k}=\frac{\partial}{\partial l_{i}} \cdot q_{k}$. Performing the differentiation in the right-hand side and expressing the scalar products via $D_{\alpha}$, we obtain the recurrence relation for the function $J$. 
Though the derivation of the IBP identities is trivial, their application to the reduction is seriously hampered by their infinite number. The problem is that it is not always clear, which identities should be used to reduce a given integral. One standard approach, which has proved to be useful, is considering the identities starting from the simplest ones and creating a database of the rules for the reduction [3]. The drawbacks of this approach are obvious: as any undirected search, this procedure is both time- and memory-consuming, which, for complicated cases, means inaccessible for computers available. The advantage of this method is its universal applicability. Another approach to the problem is to reformulate it as a problem of "division with the remainder" with respect to some ideal $[5,6,4]$. The main difficulty on this way is the derivation of the Gröbner basis of the ideal, the procedure which is not always successful [7].

The consideration presented here is based on Ref. [8]. It makes use of a simple observation that operators $O_{i k}$ form a closed Lie algebra which corresponds to the group of the linear change of variables (LCV group). The closed Lie-algebraic structure of the IBP identities is reflected in the huge redundancy of the IBP identities. This redundancy, while being harmless for not very large systems of equations, essentially slows down the Laporta algorithm. On the other hand, the same Lie-algebra structure makes the problem of IBP reduction very special case of the general problem of reduction. Therefore, the construction of the Gröbner basis seems to be too general approach, which is neither necessary nor always successful. Moreover, we demonstrate that, strictly speaking, the problem of the IBP reduction is not reduced to that of reduction modulo some ideal.

The paper is organized as follows. In Sec. 2 we introduce the operator form of the IBP reduction and formulate the problem of reduction as that of reduction modulo direct sum of definite left and right ideals. In Sec. 3 we discuss the Lie-algebraic structure of the IBP operators and its connection with the LCV group. The application of the criteria proved in [8] is also discussed in this Section.

\section{Operator representation}

Let us introduce, similar to Ref. [4], the operators $A_{\alpha}$ and $B_{\alpha}$ acting on functions in $\mathbb{Z}^{N}$ as follows

$$
\begin{aligned}
& \left(A_{\alpha} f\right)\left(n_{1}, \ldots, n_{N}\right)=n_{\alpha} f\left(n_{1}, \ldots, n_{\alpha}+1, \ldots, n_{N}\right), \\
& \left(B_{\alpha} f\right)\left(n_{1}, \ldots, n_{N}\right)=f\left(n_{1}, \ldots, n_{\alpha}-1, \ldots, n_{N}\right) .
\end{aligned}
$$

Note that these operators act on function, but not on its arguments, and should not be confused with the conventional $\mathbf{n}^{ \pm}$index shifting operators. Owing to their definition, these operators obey the following commutation relation

$$
\left[A_{\alpha}, B_{\beta}\right]=\delta_{\alpha \beta} .
$$

Our choice of the operators (2.1) is well adjusted with the notion of sectors. If $\mathbf{n}$ belongs to some sector $\boldsymbol{\theta}$, and $P$ is some polynomial operator constructed of $A_{n}, B_{m}$ then $(P J)(\mathbf{n})$ contains only the integrals of the same sector and its subsectors. While considering the IBP identities in definite sector, it is natural to introduce the ordering of monomials, constructed of $A_{k}, B_{k}$, consistent with the ordering of the integrals in this sector. E.g., as a measure of complexity of a monomial $\prod_{i} A_{i}^{\alpha_{i}} B_{i}^{\beta_{i}}$ 
in sector $\boldsymbol{\theta}$, we can use the list

$$
\left\{\sum_{i} \sigma_{i} \gamma_{i}^{-}, \sum_{i}\left(\theta_{i}-1\right) \gamma_{i}^{-}, \sigma_{1} \gamma_{1}^{-}, \ldots, \sigma_{N} \gamma_{N}^{-}, \sum_{i} \gamma_{i}^{+}, \alpha_{1}, \beta_{1}, \ldots, \alpha_{N}, \beta_{N}\right\}
$$

where $\sigma_{i}=2 \theta_{i}-1, \gamma_{i}^{ \pm}=\alpha_{i} \pm \beta_{i}$.

Using operators (2.1), we can express the IBP identities as constraints on the function $J$ having the form

$$
\left(P_{i k} J\right)(\mathbf{n})=-\int d^{\mathscr{D}} l_{1} \ldots d^{\mathscr{D}} l_{L} O_{i k} j(\mathbf{n})=0 .
$$

It follows from the definition of $O_{i k}$ that any $P_{i k}$ is a quadratic polynomial of the form

$$
P_{i k}=a^{\alpha \beta} A_{\alpha} B_{\beta}+b^{\alpha} A_{\alpha}+c
$$

where $a^{\alpha \beta}, b^{\alpha}, c$ are some coefficients (depending on $i, k$ ).

Let $\mathscr{L}$ be the left ideal generated by operators $P_{i k}$, i.e. a set, consisting of all operators, which can be represented as

$$
\sum_{i, k} C_{i k} P_{i k}
$$

where $C_{i k}$ are some polynomials of $A_{1}, \ldots A_{N}, B_{1}, \ldots, B_{N}$. This ideal has a simple meaning: for any $L \in \mathscr{L}$ the relation

$$
(L J)\left(n_{1}, \ldots, n_{N}\right)=0
$$

is a linear combination of some IBP identities. In fact, any linear combination of the IBP identities can be represented in a more specific form

$$
(L J)(1, \ldots, 1)=0,
$$

since shifting of the indices can be done by acting from the left with some powers of $A_{\alpha}$ or $B_{\alpha}$.

At first glance, the problem of reduction is equivalent to that of division with the remainder by the ideal $\mathscr{L}$, which is effectively solved by the construction of the Gröbner basis. However, there is an additional obstacle. Note that for any function $f$ of $N$ integer variables the following relation holds

$$
\left(B_{\alpha} A_{\alpha} f\right)(1, \ldots, 1)=0 \text {. (no summation) }
$$

Indeed,

$$
\left(B_{\alpha} A_{\alpha} f\right)(1, \ldots, 1)=\left(A_{\alpha} f\right)(1, \ldots, \stackrel{\alpha}{0}, \ldots, 1)=0 \times f(1, \ldots, \stackrel{\alpha}{1}, \ldots, 1)=0,
$$

where the overscript $\alpha$ denotes the position of the index. Let $\mathscr{R}$ be the right ideal generated by the elements $\left(B_{1} A_{1}\right), \ldots,\left(B_{N} A_{N}\right)$. By definition, it consists of all operators of the form

$$
R=\sum_{\alpha} B_{\alpha} A_{\alpha} C_{\alpha}
$$

where $C_{\alpha}$ are some polynomials of $A_{1}, \ldots A_{N}, B_{1}, \ldots, B_{N}$. It follows from Eq. (2.8) that

$$
(R f)(1, \ldots, 1)=0
$$


Thus, for the reduction procedure to work, we have to have an algorithm of division with the remainder by the direct sum of the left ideal $\mathscr{L}$ and the right ideal $\mathscr{R}$. That means that we have to invent the algorithm allowing the decomposition

$$
p=L+R+r
$$

where $L \in \mathscr{L}, R \in \mathscr{R}$, and $r$ is the simplest possible with respect to the ordering chosen. Even though the problem is clearly formulated, such algorithm appears to be unknown so far.

It is important to note that a naive application of consecutive reduction, first with respect to $\mathscr{L}$, next with respect to $\mathscr{R}$ does not give an acceptable reduction. The formulation presented above might give a clue of why the approaches based on Gröbner basis do not give a universal solution.

\section{LCV group and IBP identities}

Let us show now that the IBP identities possess some special features which are not taken into account in the conventional reduction procedure so far. First, we note that the operators $O_{i k}$ form a closed algebra with the commutation relations

$$
\left[O_{i k}, O_{j l}\right]=\delta_{i l} O_{j k}-\delta_{j k} O_{i l}
$$

This algebra is nothing but the algebra of the group of linear changes of variables

$$
l_{i} \rightarrow M_{i k} q_{k}
$$

The operator $O_{i k}=\frac{\partial}{\partial l_{i}} \cdot q_{k}$ corresponds to the infinitesimal transformation $l_{i} \rightarrow l_{i}^{\prime}=l_{i}+\varepsilon q_{k}$ in the sense that

$$
f\left(s_{l m}^{\prime}\right) d^{\mathscr{D}} l_{1}^{\prime} \ldots d^{\mathscr{D}} l_{L}^{\prime}=\left\{f\left(s_{l m}\right)+\varepsilon\left[\frac{\partial}{\partial l_{i}} \cdot q_{k} f\left(s_{l m}\right)\right]\right\} d^{\mathscr{D}} l_{1} \ldots d^{\mathscr{D}} l_{L}+O\left(\varepsilon^{2}\right) .
$$

Note that the so-called symmetry relations are also the consequences of the invariance of the integral under the action of some elements of this group.

We can easily check that the operators $P_{i k}$ obey the same commutation relations as the operators $O_{i k}$ :

$$
\begin{aligned}
\left(\left[P_{1}, P_{2}\right] J\right)(\mathbf{n}) & =\left(P_{1} P_{2} J\right)(\mathbf{n})-\left(P_{2} P_{1} J\right)(\mathbf{n})=\int d^{\mathscr{D}} l_{1} \ldots d^{\mathscr{D}} l_{L} O_{2} O_{1} j(\mathbf{n})-\int d^{\mathscr{D}} l_{1} \ldots d^{\mathscr{D}} l_{L} O_{1} O_{2} j(\mathbf{n}) \\
& =-\int d^{\mathscr{D}} l_{1} \ldots d^{\mathscr{D}} l_{L}\left[O_{1}, O_{2}\right] j(\mathbf{n}) .
\end{aligned}
$$

The minus sign in the last line is the same as in Eq. (2.4). 
In Ref. [8] several consequences of this algebraic structure have been outlined. The first criterion concerns the automatic search of zero sectors. According to this criterion, it is sufficient to solve the IBP identities in the corner point of the sector in order to determine whether the sector is zero or not. If the solution results in vanishing of the simplest integral of the sector, the whole sector is zero, otherwise, it is not.

Criteria II and III from Ref. [8] allow one to essentially reduce the set of the operators to be considered. Let us discuss the Criterion III of Ref. [8]. As an example, we consider the three-loop massive tadpole, shown in Fig. 1. The reduction rules for the massive tadpoles have been

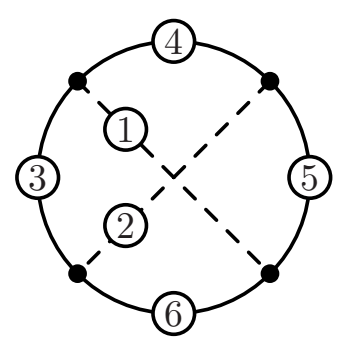

Figure 1: 3 loop tadpole topology. obtained in Ref. [9]. Thus, our example serves only as a demonstration of the method. The IBP operators corresponding to this topology have the form

$$
\begin{aligned}
P_{11}= & -\mathscr{D}+2 A_{3}+2 A_{6}+A_{1} B_{1}-A_{6} B_{2}+A_{1} B_{3}+2 A_{3} B_{3}+A_{6} B_{3}-A_{1} B_{4}+A_{6} B_{6}, \\
P_{12}= & -B_{1} A_{3}+B_{3} A_{3}+B_{4} A_{3}+2 A_{3}+2 A_{6}-A_{1} B_{1}-A_{6} B_{1}-A_{6} B_{2}+A_{1} B_{3}+A_{6} B_{3} \\
& -A_{1} B_{4}+A_{6} B_{5}, \\
P_{13}= & -B_{1} A_{3}-B_{2} A_{3}+B_{4} A_{3}+B_{6} A_{3}+2 A_{3}+2 A_{6}-A_{1} B_{1}-A_{6} B_{1}-A_{1} B_{5}+A_{6} B_{5} \\
& +A_{1} B_{6}+A_{6} B_{6}, \\
P_{21}= & -B_{1} A_{4}+B_{3} A_{4}+B_{4} A_{4}+2 A_{4}-2 A_{6}-A_{1} B_{1}+A_{2} B_{2}+A_{6} B_{2}-A_{1} B_{3}+A_{2} B_{3} \\
& -A_{6} B_{3}+A_{1} B_{4}-A_{2} B_{6}-A_{6} B_{6}, \\
P_{22}= & -\mathscr{D}+2 A_{4}-2 A_{6}+A_{1} B_{1}+A_{6} B_{1}+A_{2} B_{2}+A_{6} B_{2}-A_{1} B_{3}-A_{6} B_{3}+A_{1} B_{4} \\
& +A_{2} B_{4}+2 A_{4} B_{4}-A_{2} B_{5}-A_{6} B_{5}, \\
P_{23}= & -B_{2} A_{4}+B_{4} A_{4}+B_{5} A_{4}+2 A_{4}-2 A_{6}+A_{1} B_{1}+A_{6} B_{1}-A_{2} B_{2}+A_{2} B_{4}+A_{1} B_{5} \\
& -A_{2} B_{5}-A_{6} B_{5}-A_{1} B_{6}-A_{6} B_{6}, \\
P_{31}= & -B_{1} A_{5}-B_{2} A_{5}+B_{4} A_{5}+B_{6} A_{5}+2 A_{5}+2 A_{6}-A_{2} B_{2}-A_{6} B_{2}-A_{2} B_{3}+A_{6} B_{3} \\
& +A_{2} B_{6}+A_{6} B_{6}, \\
P_{32}= & -B_{2} A_{5}+B_{4} A_{5}+B_{5} A_{5}+2 A_{5}+2 A_{6}-A_{6} B_{1}-A_{2} B_{2}-A_{6} B_{2}+A_{6} B_{3}-A_{2} B_{4} \\
& +A_{2} B_{5}+A_{6} B_{5}, \\
P_{33}= & -\mathscr{D}+2 A_{5}+2 A_{6}-A_{6} B_{1}+A_{2} B_{2}-A_{2} B_{4}+A_{2} B_{5}+2 A_{5} B_{5}+A_{6} B_{5}+A_{6} B_{6}
\end{aligned}
$$

Taking into account the symmetry, one can consider 7 different sectors, which we choose as

$$
(1,1,1,1,1,1), \quad(1,1,0,1,1,1), \quad(0,1,1,1,1,1),
$$

$$
(1,1,0,1,0,1), \quad(0,1,0,1,1,1), \quad(0,0,1,1,1,1), \quad(0,0,0,1,1,1) .
$$

Let us introduce the notion of "sharpening" of the system of operators in a given sector. This stands for the Gauss triangularization of the above system of polynomials with respect to the most complex monomials in the system.

The sharpening of the system (3.5) in first three sectors from Eq. (3.6) immediately reveals the operators which can alway be used for the reduction in these sectors. These are $P_{33}-P_{32}, P_{11}-P_{12}$, $P_{33}-P_{32}$, respectively. 
The reduction of the topology $(1,1,0,1,0,1)$ is not that simple. The sharpening in this sector results in the following operators:

$$
\begin{gathered}
\mathscr{P}_{1}=P_{11}+P_{22}+P_{33}=-2 A_{4}+\ldots, \quad \mathscr{P}_{2}=P_{12}-P_{11}=-A_{6} B_{5}+\ldots, \\
\mathscr{P}_{3}=-P_{12}+P_{13}-P_{22}+P_{23}=-A_{4} B_{5}+\ldots, \quad \mathscr{P}_{4}=P_{11}-P_{12}+P_{33}=-A_{2} B_{5}+\ldots, \\
\mathscr{P}_{5}=P_{11}-P_{12}+P_{13}=A_{1} B_{5}+\ldots, \quad \mathscr{P}_{6}=P_{32}-P_{33}=-A_{6} B_{3}+\ldots, \\
\mathscr{P}_{7}=P_{21}-P_{22}+P_{31}-P_{32}=-A_{4} B_{3}+\ldots, \quad \mathscr{P}_{8}=P_{31}-P_{32}+P_{33}=A_{2} B_{3}+\ldots, \\
\mathscr{P}_{9}=P_{11}-P_{32}+P_{33}=-A_{1} B_{3}+\ldots
\end{gathered}
$$

Here we have shown only the leading monomials. Analyzing these monomials, we see that the rules generated by these operators fail for the integrals of one of the following forms

$$
J\left(n_{1}, n_{2}, 0,1,0, n_{6}\right), \quad J\left(1,1, n_{3}<0,1, n_{5}, 1\right), \quad J\left(1,1,0,1, n_{5}<0,1\right)
$$

Note that the maximal number of free parameters (the "dimension") of this set is only 3 . If we choose to switch to Laporta algorithm at this point, the Criterion III of Ref. [8] guarantees that we have to run in this algorithm only over this set of points (and use the above rules for the reduction). If we want to proceed further in symbolic form, we may try to construct the $S$-polynomials of our operators and to reduce them with respect to the above system of operators. In particular, $A_{6} \mathscr{P}_{4}-A_{2} \mathscr{P}_{2}=A_{6}^{2}+\ldots$. This gives us a reduction rule which is also subject to the Criterion III. Taking this rule into account, we are left with the following unexpressed integrals:

$$
J\left(n_{1}, n_{2}, 0,1,0,1\right), \quad J\left(n_{1}, n_{2}, 0,1,0,2\right), \quad J\left(1,1, n_{3}<0,1, n_{5}, 1\right), \quad J\left(1,1,0,1, n_{5}<0,1\right)
$$

This simple step reduced the dimension of our set to 2. Again, we can switch to Laporta algorithm and to run over 2-dimensional subset. Note that if we used the Laporta algorithm from the very beginning, we would have to run over 6-dimensional set of the points in a given sector.

The same analysis can be applied to the rest of the sectors. Typically, it reduces the dimension of the set of unexpressed integrals by half or more in our example and a number of the topologies considered by the author. After this analysis, one can choose between the Laporta algorithm over the rest set and more complex analysis which will be presented elsewhere.

\section{Acknowledgements}

The author thanks the organizers of the conference for stimulating him to write this contribution. This work was supported by RFBR Grant No. 07-02-00953.

\section{References}

[1] F. V. Tkachov, Phys. Lett. B 100, 65 (1981).

[2] K. Chetyrkin and F. Tkachev, Nucl. Phys. B 192, 159 (1981).

[3] S. Laporta, Int. J. Mod. Phys. A 15, 5087 (2000).

[4] A. Smirnov and V. Smirnov, JHEP 0601, 001 (2006), [hep-lat/ 0509187 ]. 
[5] O. V. Tarasov, Acta Phys. Polon. B 29, 2655 (1998).

[6] O. V. Tarasov, Nucl. Instrum. Meth. A 534, 293 (2004), [hep-ph / 0403253 ].

[7] A. Smirnov and V. Smirnov, PoS (ACAT) 085 [hep-ph/0707.3993v1].

[8] R.N. Lee, JHEP 0807, 031 (2008).

[9] D.J. Broadhurst, Z. Phys. C 54, 599 (1992). 